\title{
BMJ Open Scope, context and quality of telerehabilitation guidelines for physical disabilities: a scoping review
}

\author{
Krithika Anil (D) , ${ }^{1}$ Jennifer A Freeman, ${ }^{1}$ Sarah Buckingham, ${ }^{1}$ Sara Demain, ${ }^{1,2}$ \\ Hilary Gunn, ${ }^{1}$ Ray B Jones (D) , ${ }^{3}$ Angela Logan, ${ }^{4}$ Jonathan Marsden, ${ }^{1}$ \\ Diane Playford, ${ }^{5}$ Kim Sein, ${ }^{1}$ Bridie Kent ${ }^{3,6}$
}

To cite: Anil K, Freeman JA, Buckingham S, et al. Scope, context and quality of telerehabilitation guidelines for physical disabilities: a scoping review. BMJ Open 2021;11:e049603. doi:10.1136/ bmjopen-2021-049603

- Prepublication history and additional supplemental material for this paper are available online. To view these files, please visit the journal online. (http://dx.doi.org/10.1136/ bmjopen-2021-049603)

Received 01 February 2021 Accepted 29 July 2021

Check for updates

(C) Author(s) (or their employer(s)) 2021. Re-use permitted under CC BY. Published by BMJ.

${ }^{1}$ School of Health Professions, Peninsula Allied Health Centre, University of Plymouth, Plymouth, UK

${ }^{2}$ School of Health Sciences, University of Southampton, Southampton, UK

${ }^{3}$ School of Nursing and Midwifery, Plymouth University, Plymouth, UK

${ }^{4}$ Stroke Rehabilitation, Royal Devon and Exeter NHS Foundation Trust, Exeter, UK ${ }^{5}$ Warwick Medical School, University of Warwick, Coventry, UK

${ }^{6}$ Innovations in Health and Socia Care: A JBI Centre of Excellence, Plymouth University, Plymouth, UK

Correspondence to

Dr Krithika Anil;

krithika.anil@plymouth.ac.uk

\section{ABSTRACT}

Objective To identify the available guidance and training to implement telerehabilitation movement assessments for people (adults and children) with a physical disability, including those recovering from COVID-19.

Design Rapid scoping review.

Included sources and articles PubMed, CINAHL, Psychinfo, Cochrane, Embase, Web of Science, PEDro, UK Health Forum, WHO, National Archives and NHS England were searched using the participant-concept-context framework from 2015 to August 2020. Primary studies that recruited individuals with physical disabilities and guidance documents aimed at providers to implement movement-related telerehabilitation were included. Results 23 articles (11 primary research studies, 3 systematic reviews and 9 guidance documents) were included out of 7857 that were identified from the literature search. Two main issues were found: (1) telerehabilitation guidance (from both research studies and guidance documents) was not specific to movementrelated assessment and (2) most primary research studies provided neither guidance nor training of movementspecific assessment to practitioners. Of the COVID-19 related guidance, two articles reported COVID-19 management that only referred to identifying COVID-19 status without references to specific movement-related guidance.

Conclusions Telerehabilitation guidance and training have existed pre-COVID-19, yet the lack of specific movement-related information and provider support is surprising. This gap must be addressed to optimise effective implementation of remote assessments for those with physical disabilities.

Review registration Open Science Framework: osf.io/ vm6sp.

\section{INTRODUCTION}

Since the outbreak of the COVID-19 pandemic, health services across the world have rapidly adapted to a new way of working. They have embraced the need to use a 'digital first' approach to maintain delivery of care as best as possible, while protecting both staff and patients. ${ }^{1}$ In the field of rehabilitation, this approach is typically referred as 'telerehabilitation', defined as: the delivery
Strengths and limitations of this study

- A wide variety of articles were reviewed due to extensive searches for grey literature and no language restrictions.

- Despite no language restrictions, included articles were biased towards the English language.

- Some articles within this review may have been updated since the initial search and others have likely emerged since.

of rehabilitation services via information and communication technologies. ${ }^{2}$ This includes a broad range of services from assessment and monitoring to education and consultation. There is a general acknowledgement that, beyond the pandemic, remote delivery of services will be an integral part of everyday practice within future health and social care systems. Ensuring that the delivery of such services is both effective and equitable is pertinent, given the identified 'tsunami' of rehabilitation need ${ }^{34}$ for people with a wide range of conditions from across the lifespan (including those recovering from COVID190).$^{5}$ It is especially important to note that the practical application of telerehabilitation will not be the same across all conditions. More complicated conditions (eg, those with comorbidity) will likely require additional support than less complicated conditions. This additional consideration further demonstrates the need for comprehensive training and guidance for telerehabilitation.

Key to targeting an effective and personalised rehabilitation plan is the need for a comprehensive, detailed and valid assessment, ${ }^{6}$ with ongoing monitoring and evaluation as needs change. When the primary concerns relate to physical disability, an assessment of aspects such as dynamic posture, balance and movement is fundamental. This therefore raises important questions related 
to how these assessments can be undertake remotely, while ensuring they are both safe and effective. For instance, is it possible to adequately and safely assess the balance of an individual who is falling/at risk of falls? Or the source of shoulder pain? Or the relative impact of spasticity and weakness on movement? Do the additional practical challenges such as slow internet speeds and static and restricted camera angles mean that an assessment of dynamic movement is too restricted to inform management plans? Are the clinical skills required for this type of remote assessment different to those used for face-toface assessment? And if so, is there specific guidance and training related to these aspects of remotely based physical assessments to assist practitioners with implementing this effectively and efficiently? It was our observation that questions such as these have been raised by rehabilitation organisations, professional networks and individual practitioners and require urgent answers, hence the need for this rapid scoping review.

This scoping review formed part of an overall research project aimed at developing and evaluating a training package to support the remote assessment and management of people with physical disability (UK RI-NIHR, MRC, COVID-19: MR/V021060/1). It was not appropriate to involve patients or the public in the design, or conduct, or reporting of this research.

\section{Review question and objectives}

What guidance and training is available to implement telerehabilitation movement assessment for people (children and adults) with a physical disability, including those recovering from COVID-19? The review objectives were to identify:

- The contexts and types of telerehabilitation assessments delivered in a health and social care setting for people with a physical disability (including those recovering from COVID-19).

- Any specific guidance and/or training available for delivering telerehabilitation movement-related assessments.

- Specific recommendations regarding movementrelated assessments for effective implementation of telerehabilitation guidance.

\section{METHODS}

\section{Type of review}

This scoping review used the Joanna Briggs Institute (JBI) approach. ${ }^{7}$ This approach seeks to explore the breadth or extent of the literature, map and summarise the evidence and inform future research. ${ }^{8}$ The Preferred Reporting Items for Systematic Reviews and MetaAnalyses (PRISMA) checklist for scoping reviews was used for the review (see online supplemental material 1 titled 'SM - PRISMA Statement for Scoping Reviews'). There is no registration database specific for scoping reviews, thus the review was uploaded to Open Science Framework: osf.io/vm6sp.p9.
Patient and public involvement

No patients involved.

\section{Inclusion criteria}

The inclusion criteria were developed using the participant-concept-context framework. ${ }^{9}$

\section{Participants}

The review considered articles that included individuals of all ages with a physical disability. Physical disability was defined as any physical condition that affects a person's mobility, physical capacity, stamina or dexterity. The review included studies of participants with a physical disability who had any duration and severity of physical disability. There was no age restriction. Guidance and policy documents aimed at undertaking telerehabilitation with individuals with physical disabilities were also included.

\section{Concept}

Studies and policies that examined or described guidance and training regarding implementation of telerehabilitation movement assessments were considered. Guidance included checklists, tools, outcome measurement batteries, protocols, guidelines, risk assessments, exemplars and appraisals. Training included interactions, communications and resources with the aim to improve and/or inform the process of delivering telerehabilitation. Guidance and training must have involved movement evaluation; that is, the evaluation of how people move around, perform and function. This included selfadministered and practitioner administered telerehabilitation. Studies and policies that did not involve movement evaluation were excluded.

\section{Context}

This review considered studies that provided guidance and training in health and social care contexts; that is, telerehabilitation must have been provided by a practitioner, system or institute.

\section{Types of literature}

The following study designs were included: randomised controlled trials (RCTs), non-RCTs, quasiexperimental, prestudies and poststudies, case studies, observational studies, systematic reviews and qualitative studies. Grey literature regarding policies from recognised UK-based national and international institutes were also considered. Opinion pieces, non-systematic literature reviews and other grey literature were excluded. No language restrictions were implemented.

\section{Date restriction}

Publications from 2015 to August 2020 were included in this review because technologies and related-guidance older than this period were considered out of date due to the rapid speed of technology development in areas such as robotics, artificial intelligence and telepresence. ${ }^{1011}$ 

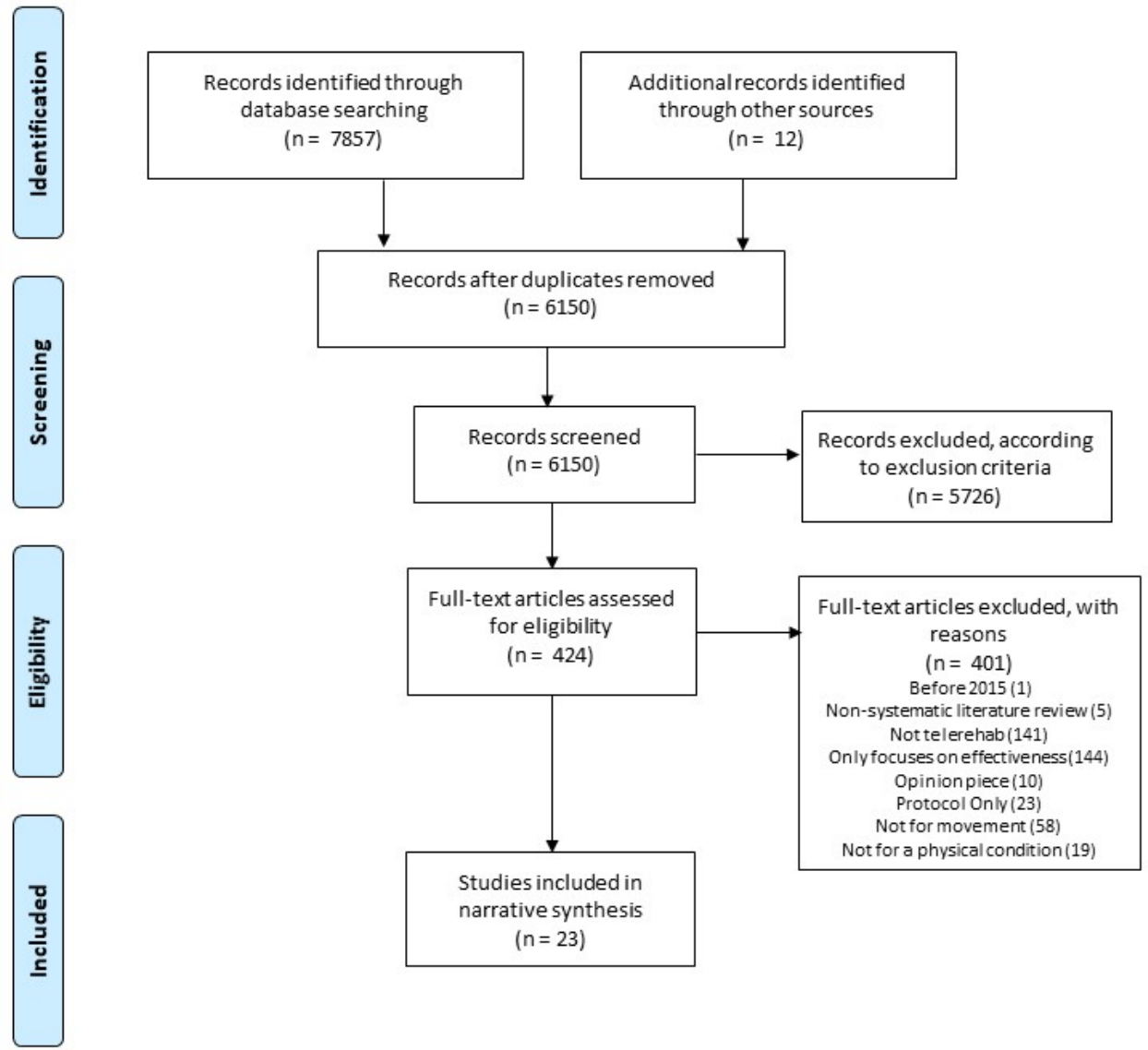

Figure 1 PRISMA flow diagram that charts the study identification process. PRISMA, Preferred Reporting Items for Systematic Reviews and Meta-Analyses.

\section{Search strategy}

An initial limited search of PubMed and CINAHL was undertaken to estimate the volume of relevant literature and to identify key words to assist in developing search terms. A second search using the developed search terms was undertaken (supported by a review-specialist librarian) and adapted across each included information source (PubMed, CINAHL, PsychInfo, Cochrane, Embase, Web of Science, PEDro, UK Health Forum, WHO, National Archives and NHS England). Due to the rapid nature of this review, study authors were not contacted for further information in cases of ongoing or uncompleted studies. Please see supplementary materials (online supplemental materials 2 and 3) for search strategy terms.

\section{Study selection}

Following the search, all identified references were imported into Endnote ${ }^{12}$ (a reference management software). After removal of duplications within EndNote, references were uploaded to the online Rayyan tool ${ }^{13}$ (a review organisation tool). Titles and abstracts were screened for assessment against the inclusion criteria for the review. The full text of potentially eligible studies were retrieved and assessed in detail against the inclusion criteria. Full-text studies that did not meet the inclusion criteria were excluded and are displayed in a PRISMA flow chart (see figure 1). Any disagreements that arose between the reviewers were resolved through discussion. As this review is a rapid scoping review, no quality or risk of bias assessment was undertaken.

\section{Data extraction}

Data extraction of included studies was conducted by the review team. Data were charted using a customised Excel spreadsheet. The data extracted specific information pertaining to the review objectives and key information for each article were charted as per JBI Guidance. ${ }^{7}$ This included telerehabilitation contexts, types of telerehabilitation, guidance and training, and any recommendations for implementing telerehabilitation. Any disagreements between reviewers were resolved through discussion.

\section{Data synthesis}

The narrative synthesis of the findings from included studies, policies and guidance documents was structured according to the review objectives. Findings were presented narratively, aided by appropriate tables and figures. Recommendations in the literature were analysed using descriptive qualitative analysis ${ }^{14}$ to identify common themes. Only recommendations focusing on clinical implementation or technological improvements were analysed (recommendations for future research within the included studies were not analysed). 
Mentions of the 'provider' refer to the individual providing the telerehabilitation, such as health or social care practitioners. Mentions of the 'client' refer to the individual receiving the telerehabilitation, that is, the patient.

\section{RESULTS}

Twenty-three articles were included in the scoping review analysis (figure 1; see table 1 for article characteristics). Eleven ${ }^{15-25}$ of the included articles were original research studies, and three were systematic review studies. ${ }^{26-28}$ The remaining nine ${ }^{29-37}$ were guidance documents produced by health institutions or health experts. Guidance documents are highlighted in grey and systematic review articles are highlighted in yellow in table 1 . Eleven articles were specified for adults ${ }^{15-19} 21-242627$ ( $\geq 18$ years), one article was specified for children ${ }^{20}$ ( $\leq 18$ years), and 11 articles did not specify age. ${ }^{25}$ 28-37

The 14 included research studies and systematic reviews had the following general aims: one study assessed use and perceptions of telerehabilitation ${ }^{15}$; one study examined costs of implementing telerehabilitation ${ }^{16}$; one study assessed the development of a telerehabilitation technology ${ }^{17}$; three studies systematically reviewed telerehabilitation literature ${ }^{26-28}$; four studies assessed the feasibility of a telerehabilitation technology ${ }^{18-21}$; and four studies examined the effectiveness of the telerehabilitation technology. ${ }^{22-25}$

\section{Contexts}

Client setting was reported in 13 of the 23 articles, where only one article did not include a home setting and instead only used general clinical sites ${ }^{20}$ (table 2). The client support environment (ie, who was with the client) was reported in 13 articles (table 2), where most clients were alone $(n=5)$. Only one article specifically reported that carers were present during the consultation or intervention. Table 3 shows the wide range of providers using telerehabilitation in people with physical disability, most being a physiotherapist. Of note, all non-qualified assistants were accompanied by a qualified health professional throughout the telerehabilitation sessions. This article ${ }^{20}$ described the development and preliminary usability, from the provider's perspective, of a tablet-based interactive movement tool. Provider setting was reported in six articles, and all were telerehabilitation technologies used by providers in secondary care (eg, an outpatient clinic). ${ }^{171921-2325}$

\section{Presence of COVID-19}

Only articles published in 2020 $16{ }^{16} 23$ 29-37 $(\mathrm{n}=12)$ were considered for COVID-19 specific information. Of these, two articles refer to COVID-19 related management: guidance documents developed by AHPScot ${ }^{29}$ and the Health and Social Care Board Northern Ireland. ${ }^{30}$ The COVID-19 related recommendations from these guides are detailed in the Recommendations section. It is noteworthy that these guidance documents refer to COVID-19 in general and are not specifically related to telerehabilitation use in people with movement impairment, despite these documents generally considering the use of telerehabilitation for this purpose. Teleswallowing $\mathrm{Ltd}^{31}$, Middleton et $a l^{16}$ and two articles from the Charted Society of Physiotherapy ${ }^{33} 34$ also refer to COVID-19 but only state that telerehabilitation is useful to decrease the spread of COVID-19; these articles do not refer to management of patients with COVID-19.

\section{Types of telerehabilitation}

Table 4 shows the aims of the telerehabilitation interventions examined in the primary research studies. Nine guides, ${ }^{29-37}$ three systematic reviews ${ }^{26-28}$ and two primary studies $^{18}{ }^{25}$ are excluded from table 4 as they did not evaluate a specific telerehabilitation technology. Most (five of the nine studies) telerehabilitation interventions aimed to assist in management of a movement condition; however, some cited additional aims such as optimising adherence, ${ }^{15}$ improving quality of life,${ }^{24}$ monitoring and assessing client satisfaction. ${ }^{23}$ One intervention provided training in using a wheelchair. ${ }^{22}$

We aimed to summarise the various platforms used for delivery of telerehabilitation, but this proved difficult, as some articles did not have sufficient detail. For example, one article ${ }^{16}$ reports a tablet app but does not specify whether video was involved or whether it just used a chat/telephone function. Accepting these limitations, the most common delivery system appeared to be via video (eg, video call, Skype, video-recording, etc), followed by apps (on tablets or mobiles) and telephone calls. Fifteen of the telerehabilitation platforms were synchronous $^{16} 171920$ 22-24 28-31 33 35-37 (ie, in real-time) and seven platforms used both synchronous and asynchronous ${ }^{15} 182126273234$ (ie, not in real-time) methods. Only one article used only an asynchronous platform where providers assessed physical ability from a video-recording of the client. ${ }^{25}$

Of the 11 primary research studies, five $\mathrm{e}^{15 \text { 17-1922 }}$ specified neither number of sessions nor session duration, one specified just number and one specified just duration. The number of sessions ranged from 1 to $36(\mathrm{n}=2$ for 1 session $^{20}{ }^{25} ; \mathrm{n}=1$ for 16 sessions $^{23}$; $\mathrm{n}=1$ for 28 sessions $^{21}$; $\mathrm{n}=2$ for 36 sessions). ${ }^{16} 24$ Three studies included $60 \mathrm{~min}$ sessions, ${ }^{20} 2324$ one included $50 \mathrm{~min}$ sessions ${ }^{16}$ and one included $100 \mathrm{~min}$ sessions. ${ }^{21}$ The variability seen here is reflective of that seen in face-to-face rehabilitation sessions.

\section{Guidance}

Specific guidance on how to use telerehabilitation technology was supplied to providers in 12 articles, ${ }^{20} 22$ 25 29-37 of which three were studies ${ }^{20225}$ and the remaining were guidance documents. ${ }^{29-37} \mathrm{~A}$ guidebook or manual was provided in 10 articles, ${ }^{20} 222529-3133-3537$ while two articles provided a checklist. ${ }^{32}{ }^{36}$ No guidance was supplied in 


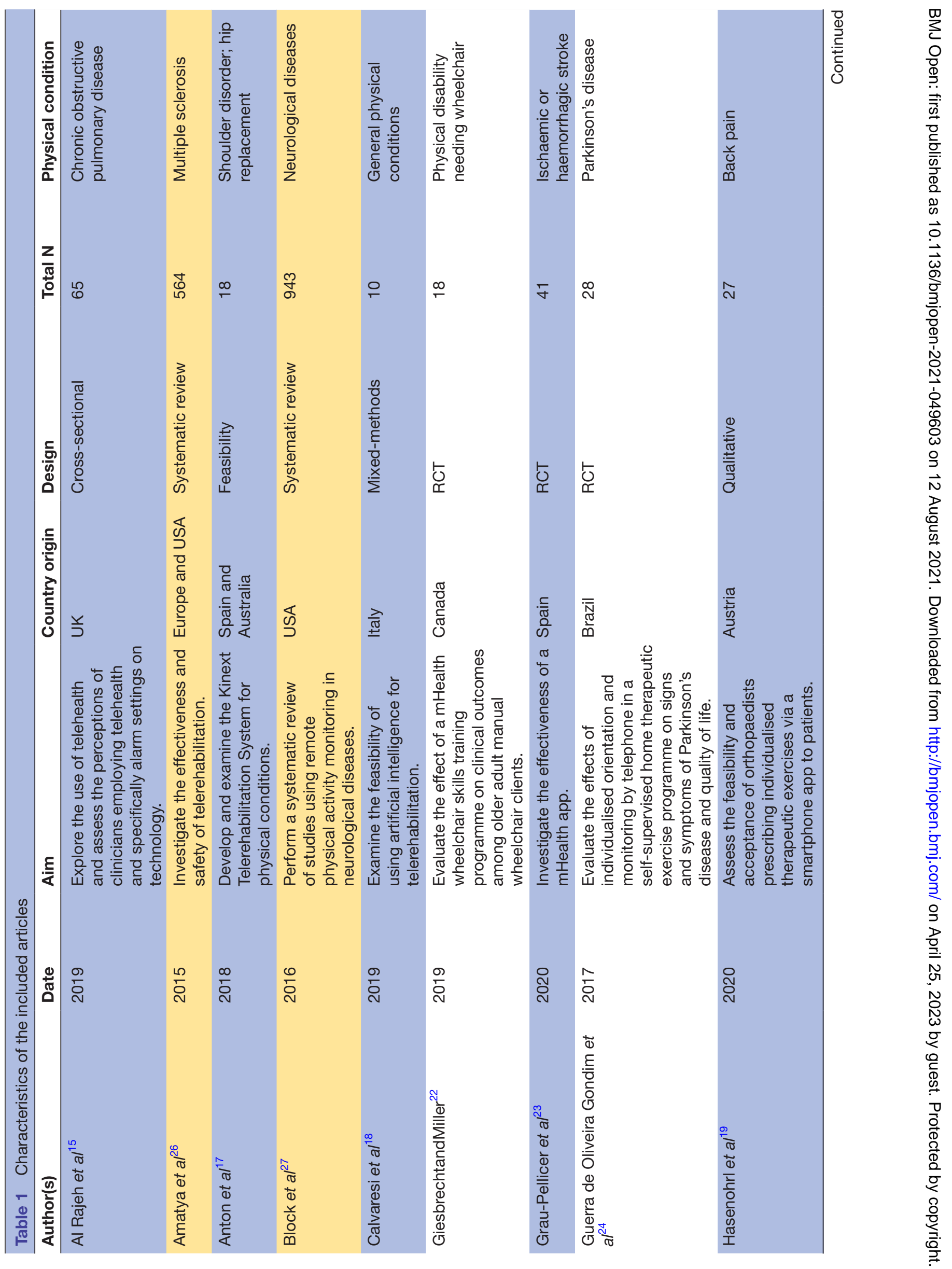




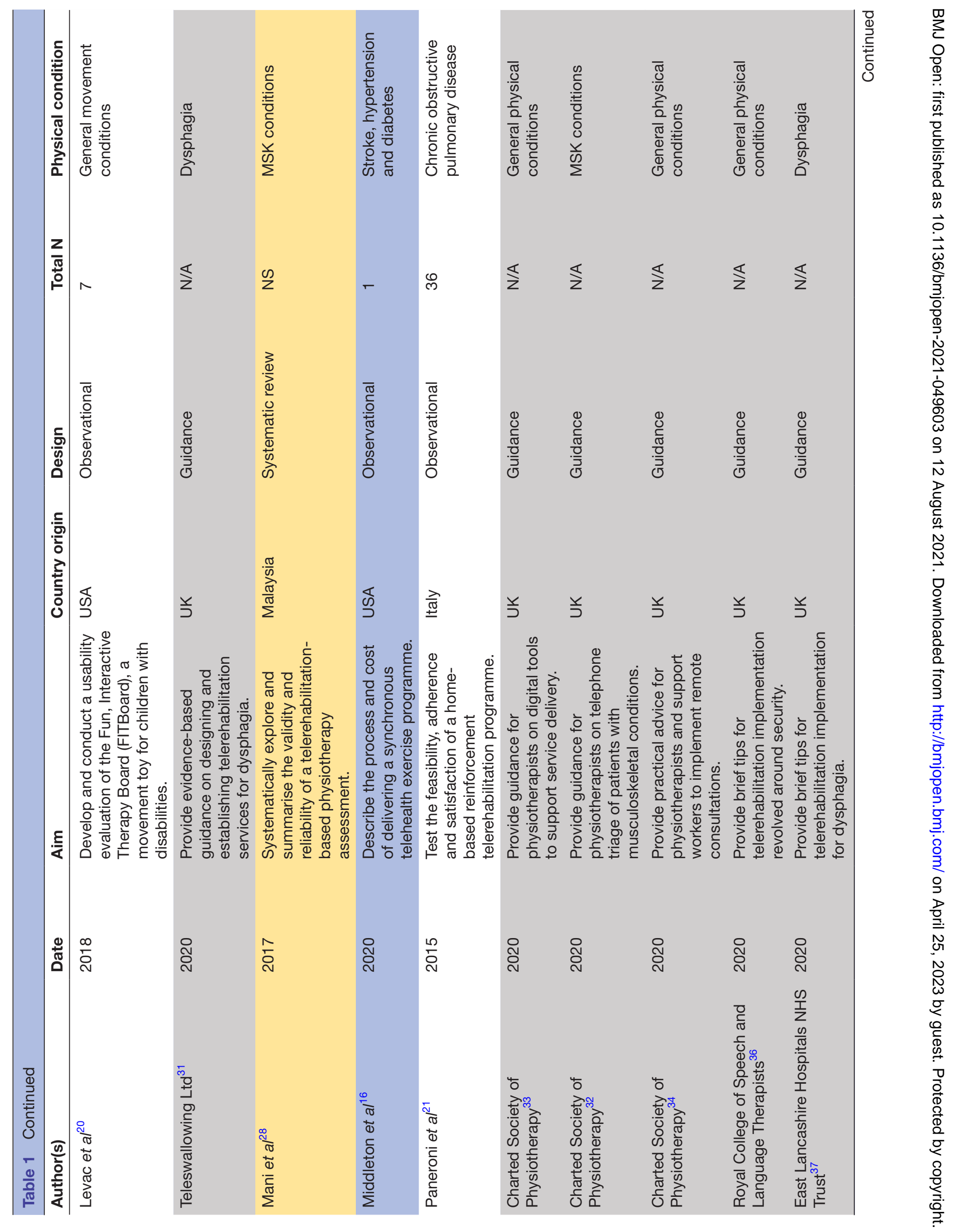




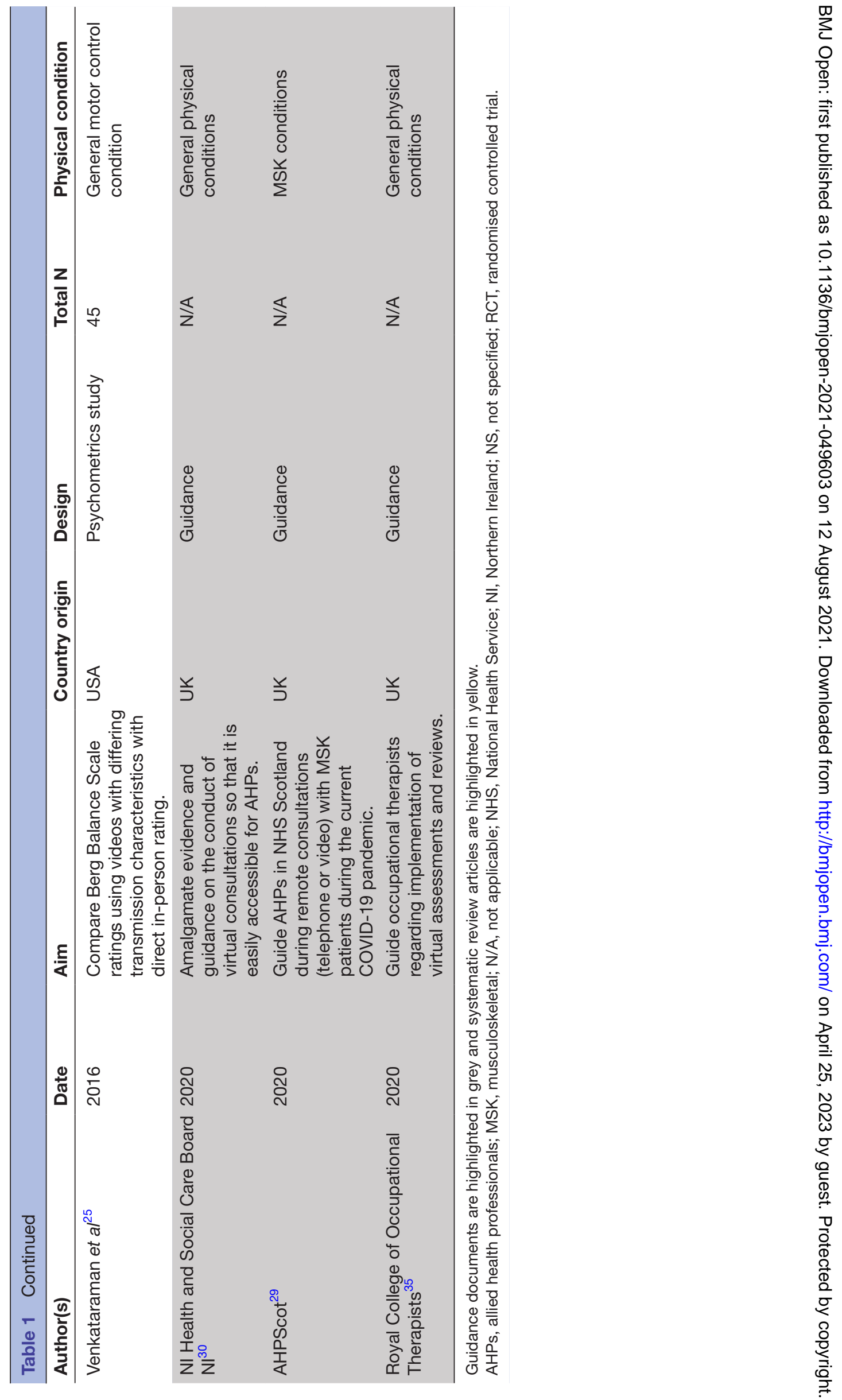


Table 2 Cross-tabulation of client support and client setting information extracted from included articles

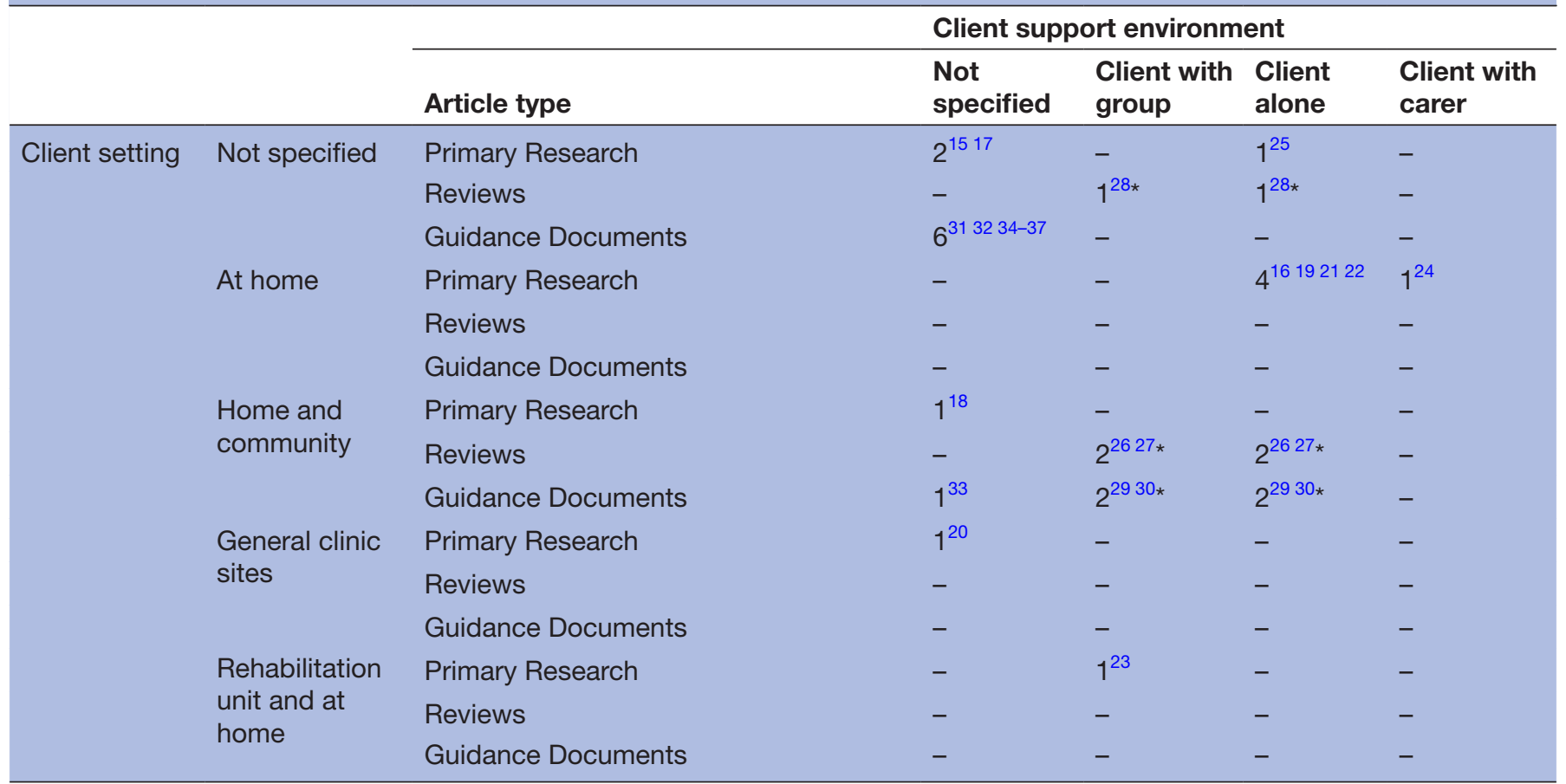

${ }^{*}$ Five articles (three reviews ${ }^{26-28}$ and two guidance documents ${ }^{29}{ }^{30}$ ) had participants complete the telerehabilitation alone and in a group; these articles have been added to both the 'Client was alone' and 'Client with group' categories.

seven articles, 151619212324 and five studies did not mention whether guidance was provided or not. ${ }^{17} 1826-28$

\section{Training}

Guidance documents and systematic reviews were not included in training analysis as these articles had no participants to whom to supply training. Specific training on how to use telerehabilitation technology was supplied to providers in 4 out of 11 primary research studies. ${ }^{16} 202225$ The training was provided in-person in three of these studies, ${ }^{162022}$ while the remaining study provided training via a video tutorial..$^{25}$ No training was supplied in five studies, ${ }^{15} 19212324$ and two studies did not mention training. ${ }^{1718}$

\section{Recommendations}

Six themes and 11 subthemes were identified within the recommendations: administrative advice, challenges, governance, social support, technology improvements and COVID-19 (see table 5). Recommendations were also analysed to extract procedural guidance that providers can perform in preparation for, during and after a

Table 3 List of telerehabilitation administrators extracted from included articles

\begin{tabular}{|c|c|c|c|}
\hline Telerehabilitation administrator* & $\mathbf{N}+$ & $\mathbf{N} \ddagger$ & N§ \\
\hline Physiotherapist & $9^{15-18202123-25}$ & $1^{28}$ & $1^{34}$ \\
\hline Occupational therapist & $3^{202225}$ & - & $1^{35}$ \\
\hline Nurse & $2^{1521}$ & - & - \\
\hline Speech and language therapist & - & - & $2^{3137}$ \\
\hline AHPs & - & - & $2^{2930}$ \\
\hline Not specified & $1^{36}$ & $1^{26}$ & - \\
\hline Assistants & $2^{1625}$ & - & - \\
\hline Physiologist & $1^{15}$ & - & - \\
\hline
\end{tabular}

*Telerehabilitation administrators overlap in articles; therefore, total $\mathrm{N}$ does not sum to total included articles.

$\dagger \mathrm{N}$ for primary research studies.

$\ddagger \mathrm{N}$ for systematic review studies.

$\S N$ for guidance documents. 
Table 4 List of telerehabilitation aims and platforms reported in the included articles

\begin{tabular}{lll}
\hline Telerehabilitation aim & N & References \\
\hline MCM & 5 & $161719-21$ \\
MCM and adherence & 1 & 15 \\
MCM and quality of life & 1 & 24 \\
MCM and client satisfaction & 1 & 23 \\
Training for assistive device use & 1 & 22 \\
\hline
\end{tabular}

MCM, movement condition management.

telerehabilitation session. This guidance is summarised in supplementary materials (online supplemental material 4) as it extended the review beyond its key message.

\section{Administrative recommendations}

Three articles ${ }^{303236}$ provided advice on administrative duties that providers should perform when conducting telerehabilitation. The advice revolved around four subthemes:

1. General advice involved general administrative duties such as maintaining record-keeping standards and identifying the specific resources a provider will need when engaging in telerehabilitation (eg, number of

Table 5 Summary of themes identified during qualitative analysis of article recommendations

\begin{tabular}{|c|c|}
\hline Analysis themes & Description \\
\hline $\begin{array}{l}\text { Administrative } \\
\text { recommendations } \\
\text { General } \\
\text { Client specific } \\
\text { Technology specific } \\
\text { Data security }\end{array}$ & $\begin{array}{l}\text { Advice revolved around } \\
\text { administrative duties, such as } \\
\text { record keeping, technology } \\
\text { maintenance or data security. }\end{array}$ \\
\hline $\begin{array}{l}\text { Challenges } \\
\text { Care provision } \\
\text { Social factors } \\
\text { Technology issues }\end{array}$ & $\begin{array}{l}\text { Challenges that providers may } \\
\text { encounter when conducting } \\
\text { telerehabilitation services. }\end{array}$ \\
\hline Governance & $\begin{array}{l}\text { Ethical advice, such as client } \\
\text { consent and/or assessing } \\
\text { confidentiality. }\end{array}$ \\
\hline $\begin{array}{l}\text { Support mechanisms } \\
\text { Providers } \\
\text { Carers } \\
\text { Provider assistants } \\
\text { Non-clinical staff }\end{array}$ & $\begin{array}{l}\text { Advice that emphasised the } \\
\text { important role of support from } \\
\text { various sources involved in } \\
\text { providing telerehabilitation care to } \\
\text { the client. }\end{array}$ \\
\hline $\begin{array}{l}\text { Technology } \\
\text { improvements }\end{array}$ & $\begin{array}{l}\text { Suggestions that may improve } \\
\text { the use and function of a } \\
\text { telerehabilitation technology. }\end{array}$ \\
\hline COVID-19 & $\begin{array}{l}\text { Actions providers are advised to } \\
\text { conduct to address COVID-19 } \\
\text { risks. }\end{array}$ \\
\hline
\end{tabular}

sessions needed, appropriate length of session considering technology set-up, clinical outcome measures needed, etc).

2. Client-specific advice involved duties that required interactions with the client, such as documenting all client conversations and noting care that would have been provided if the intervention was delivered face to face but was not done so due to the restrictions of telerehabilitation.

3. Technology-specific advice involved ensuring technology was up to date and that the latest version of any software was being used.

4. Data security emphasised that providers must minimise identifiable information that is shared digitally, ensure that all actions comply with information governance and only use technology recognised by the institute through which they provide their care services.

\section{Challenges}

Six articles $^{15} 1923252830$ highlighted challenges when conducting telerehabilitation, which involved three subthemes:

1. Care provision considers the resources needed for telerehabilitation, highlighting that some clinical assessments may be too complicated to conduct effectively at a distance and that, if this complication is not recognised by inexperienced telerehabilitation providers, the quality of care provided may be reduced.

2. Social factors identified social challenges, where unsatisfactory video etiquette, minimal rapport building and socioeconomic disadvantages have the potential to reduce the quality of care provided via telerehabilitation.

3. Technology issues relate to technology failures or interruptions, such as inadequate internet connection, low camera/audio quality and false alarms (on wearable devices) that result in a reduced quality of care.

\section{Governance}

Six articles ${ }^{29} 30$ 34-37 provided recommendations relating to governance issues. The guidance highlighted that all clients must be informed about the following: telerehabilitation is voluntary; potential risks when engaging in telerehabilitation; what will happen with their data; and what reasonable actions they should take to ensure the security of their technology. This theme also emphasised the importance of consent, where informed consent must be taken explicitly from clients when video/audio recording or taking screen shots. If required, clients must be asked whether they consent for their trusted others (eg, carer or family) to attend the session with them. However, one article $^{30}$ stated that consent was implied when clients enter the telerehabilitation session.

\section{Support mechanisms}

Six articles 2124283137 recommended support mechanisms to be set in place for clients when providing telerehabilitation. This support involved four subthemes: 
1. Providers to offer support beyond the main goals of telerehabilitation, such as reassurance to clients who are anxious using technology for their rehabilitation.

2. Carers to offer clients in-person support during delivery of telerehabilitation sessions. For instance, provision of physical assistance to safely enable the client to achieve correct posture or movement or to assist the client with using the technology, such as positioning of the camera.

3. Provider assistants to assist qualified staff by visiting clients in person to assist telerehabilitation, such as undertaking outcome measures face to face, when required and safe to do so.

4. Non-clinical staff, such as administrators and senior management, to provide essential support in successful coordination and implementation of telerehabilitation, emphasising the importance of effective communication between support staff and providers.

\section{Technology improvements}

Eight articles $^{15}$ 17-20 26-28 recommended technology improvements for telerehabilitation purposes. For instance, communications should be synchronous, and technology must have a client-friendly interface that satisfies both the client and the provider. Wearable technologies relevant to posture and movement (eg, to measure steps or heart rate) must be precise. Technology would especially benefit from improved monitoring capabilities, such as real-time movement tracking and activity adherence monitoring. If technology provided smart data (eg, real-time measures of muscle strength), it should be accompanied by useful and actionable information. Reminders to engage in self-care activities would be useful for clients during their rehabilitation period. A built-in anatomical map to assist clients in showing the location of pain, tenderness or other sensations was also suggested.

\section{COVID-19}

Two articles (both guidance documents) ${ }^{29} 30$ made recommendations to address COVID-19 concerns. When establishing whether the client needs telerehabilitation or face-to-face care, professionals were advised to identify the client's COVID-19 status. Suggested identifications included: tested positive and self-isolating, has symptoms and self-isolating, has come into contact with someone who tested positive and is now self-isolating, shielding or no current cautions outside of government restrictions. Of note, this is an area of rapid expansion in the literature.

\section{DISCUSSION}

The 23 articles included in this rapid scoping review revealed two main gaps in the literature: (1) telerehabilitation guidance (from research studies and guidance documents) is not specific to movement-related rehabilitation despite articles stating this explicitly as their aim and (2) neither guidance nor training were given to providers in most research studies evaluating a telerehabilitation technology.

\section{The lack of specific guidance}

The vast majority of the existing recommendations relate predominately to communication or governance elements of telerehabilitation and lack specific guidance about issues pertinent to movement impairment. Several elements that are key to effectively implementing telerehabilitation for people with physical disability are missing. When assessing movement remotely, one would expect guidance on ensuring patient safety, triaging patients and specific steps to decide whether a patient needs faceto-face care. More importantly, the identified guidance does not effectively address the limitations of undertaking physical assessments remotely, for example, if the patient is unable to move their body themselves if their movement is inadequate for assessment. Remote physical examinations are complex and need specific guidance on implementation; this is reflected in recent research that highlights physical examinations as one of the main challenges for remote assessments. ${ }^{389}$ There was also minimal information on telerehabilitation related to COVID-19. This is unsurprising given the novelty of this infection. Overall, current telerehabilitation guidance for physical disabilities focuses on general telehealth guidance rather than being specific to movement impairments. Although issues such as data security, video etiquette and organisation are important, they are not enough to assist providers in effective telerehabilitation implementation for people with movement impairments.

\section{The minimal focus on providers}

Of the 11 included primary studies and three systematic reviews, only three 202225 provided guidance to providers. Similarly, only four research studies ${ }^{162022} 25$ described the provision of training to providers. Neglecting providers' skills in using technology effectively (ie, digital literacy) potentially undermines the usefulness of any telerehabilitation technology that was evaluated. ${ }^{40}{ }^{41}$ Furthermore, studies mainly report the clients' and not the providers' context of using telerehabilitation (eg, whether the provider was from primary, secondary or tertiary care). The generalisability of the telerehabilitation technology examined in the study is thus unclear, as certain technologies may only be effective in particular contexts. ${ }^{42}$ Future studies should carefully consider providers' digital literacy and context to better understand the effectiveness of telerehabilitation technology. Digital literacy and context are not the only important factors for telerehabilitation implementation. Healthcare providers need appropriate organisational infrastructures (eg, effective IT support) and a sufficient workflow integration to effectively implement technology. ${ }^{43}$ They also reported that an easy-to-use interface is important, which aligns with the review's finding of needing a friendly client interface for technological improvements. 


\section{Digital equity}

Although digital equity was not part of this review's objectives, it is important to address. The included articles provide information based on the assumption that all clients (and providers) have both access and skills to engage in technology for remote assessments. Many of those currently digitally excluded are likely to become increasingly neglected from remote health services, thereby exacerbating existing health inequalities. ${ }^{44}$ Future studies should acknowledge equity of access when examining telerehabilitation technologies. Future guidance documents should provide information for when clients do not have access to (or decline to use) telerehabilitation.

\section{Comparisons with other studies}

To our knowledge, this is the first scoping review of telerehabilitation guidance and training for assessing physical disabilities within daily clinical practice. Previous reviews mostly examine effectiveness within the context of research studies and conclude that further provider and client training is needed to adequately implement telerehabilitation for physical assessments. ${ }^{45}{ }^{46}$ These studies conclude that telerehabiltiation technology has potential to deliver effective physical disability care; the current state of the art is insufficient for accurate remote movement assessment. ${ }^{47}$ Our review confirms the need for further provider training and additionally emphasises the lack of specific movement-related guidance.

\section{Study limitations}

This scoping review has several limitations. First, many telerehabilitation guidance documents for physical disabilities are likely unpublished. Although this review conducted an extensive search within grey literature sources, guidance documents and other relevant studies have likely been missed. Second, the search strategy was biased towards the English language. Despite no language restrictions during the search and the use of language translation software, relevant articles have likely been missed. This review also included a date restriction, where no articles prior to 2015 were included in the literature search in order to exclude outdated technological information. Yet, it should be noted that this may have also excluded some relevant aspects on guidance and training. Finally, this review was restricted by its rapid, cross-sectional approach. The authors acknowledge that some articles within this review may have been updated since the initial search and others have likely emerged since. This review may serve as a foundation for future research and development of guidance documents to provide information that addresses the gaps identified above.

\section{CONCLUSION}

There is a clear need for specific telerehabilitation guidance for physical disabilities, particularly for effective practice of remote physical assessments. Studies examining telerehabilitation technologies should deliver guidance and training to providers as well as document the context of providers if those technologies are to be effectively implemented. The catalyst that is the COVID-19 pandemic has forced the uptake of remote rehabilitation services, which are likely to persist beyond the pandemic. The development and maintenance of efficient telerehabilitation will require detailed guidance and active performance monitoring for ongoing improvement of existing guidance, without which remote physical assessments may result in suboptimal management.

Twitter Krithika Anil @pain_is_weird, Ray B Jones @rjonesplymouth, Angela Logan @logan_angie and Bridie Kent @BridieKent

Acknowledgements We would like to sincerely thank Chris Johns from the University of Plymouth, who provided library support during the initial phase of this scoping review, and particularly assisted in developing the search strategy. Thanks to Sarah Chatfield, who undertook the initial limited search of the literature.

Contributors KA planned and conducted the literature search, organised the review team, analysed and reported the results and produced the initial manuscript. JAF conceptualised the study idea and supervised study processes. All authors reviewed the articles from the literature search, extracted data, contributed to data interpretation and critically evaluated the manuscript. The corresponding author, KA, attests that all listed authors meet authorship criteria and that no others meeting the criteria have been omitted.

Funding This work was supported by UKRI-NIHR (MRC Section), Covid-19; Reference MR/V021060/1.

Disclaimer The funder had no role in study design, data collection, data analysis or writing of the report.

Competing interests None declared.

Patient consent for publication Not required.

Ethics approval This study is a scoping literature review and did not involve participant data collection. Therefore, ethical approval was not required.

Provenance and peer review Not commissioned; externally peer reviewed. Data availability statement No data are available.

Supplemental material This content has been supplied by the author(s). It has not been vetted by BMJ Publishing Group Limited (BMJ) and may not have been peer-reviewed. Any opinions or recommendations discussed are solely those of the author(s) and are not endorsed by BMJ. BMJ disclaims all liability and responsibility arising from any reliance placed on the content. Where the content includes any translated material, BMJ does not warrant the accuracy and reliability of the translations (including but not limited to local regulations, clinical guidelines, terminology, drug names and drug dosages), and is not responsible for any error and/or omissions arising from translation and adaptation or otherwise.

Open access This is an open access article distributed in accordance with the Creative Commons Attribution 4.0 Unported (CC BY 4.0) license, which permits others to copy, redistribute, remix, transform and build upon this work for any purpose, provided the original work is properly cited, a link to the licence is given, and indication of whether changes were made. See: https://creativecommons.org/ licenses/by/4.0/.

ORCID iDs

Krithika Anil http://orcid.org/0000-0002-8027-1665

Ray B Jones http://orcid.org/0000-0002-2963-3421

\section{REFERENCES}

1 Peek N, Sujan M, Scott P. Digital health and care in pandemic times: impact of COVID-19. BMJ Health Care Inform 2020;27:e100166.

2 Brennan D, Tindall L, Theodoros D, et al. A blueprint for telerehabilitation guidelines. Int J Telerehabil 2010;2:31-4.

3 Charted Society of Physiotherapy. Rehabilitation and Covid-19 - CSP policy statement, 2020. Available: https://www.csp.org. 
uk/professional-clinical/improvement-innovation/communityrehabilitation/rehab-covid-19-policy-statement

4 Phillips M, Turner-Stokes L, Wade D. Rehabilitation in the wake of Covid-19-a Phoenix from the ashes. British Society of Rehabilitation Medicine 2020 https://www.cmsuk.org/executive-summary-ofrehabilitation-in-the-wake-of-covid-19-a-phoenix-from-the-ashes

5 Wade DT. The future of rehabilitation in the United Kingdom National health service: using the COVID-19 crisis to promote change, increasing efficiency and effectiveness. London, England: SAGE Publications Sage UK, 2020.

6 Wade DT. What attributes should a specialist in rehabilitation have? seven suggested specialist capabilities in practice. London, England: SAGE Publications Sage UK, 2020: 34. 995-1003.

7 Aromataris E, Munn Z. Chapter 1: JBI systematic reviews. In: Aromataris E, Munn Z, eds. JBI manual for evidence synthesis. Adelaide, Australia: JBI, 2020

8 Tricco AC, Lillie E, Zarin W, et al. A scoping review on the conduct and reporting of scoping reviews. BMC Med Res Methodol 2016;16:15

9 Peters MDJ, Marnie C, Tricco AC, et al. Updated methodological guidance for the conduct of scoping reviews. JBI Evid Synth 2020;18:2119-26.

10 Meyerson B. Top 10 emerging technologies of 2015 world economic forum, 2015. Available: https://www.weforum.org/agenda/2015/03/ top-10-emerging-technologies-of-2015-2

11 Wood L. Global telepresence robots market (2020 to 2025) - growth, trends, and forecast business wire, 2020. Available: https://www. businesswire.com/news/home/20201005005668/en/GlobalTelepresence-Robots-Market-2020-to-2025-Growth-Trends-andForecast-ResearchAndMarkets.com

12 Endnotex9Philadelphia, USReuters T, 2013Clarivate. Available: https://endnote.com/product-details

13 Ouzzani M, Hammady H, Fedorowicz Z, et al. Rayyan-a web and mobile APP for systematic reviews. Syst Rev 2016;5:210.

$14 \mathrm{Kim} \mathrm{H}$, Sefcik JS, Bradway C. Characteristics of qualitative descriptive studies: a systematic review. Res Nurs Health 2017;40:23-42.

15 Al Rajeh A, Steiner MC, Aldabayan Y, et al. Use, utility and methods of telehealth for patients with COPD in England and Wales: a healthcare provider survey. BMJ Open Respir Res 2019;6:e000345.

16 Middleton A, Simpson KN, Bettger JP, et al. COVID-19 pandemic and beyond: considerations and costs of telehealth exercise programs for older adults with functional impairments living at Home-Lessons learned from a pilot case study. Phys Ther 2020;100:1278-88.

17 Anton D, Berges I, Bermúdez J, et al. A Telerehabilitation system for the selection, evaluation and remote management of therapies. Sensors 2018;18. doi:10.3390/s18051459. [Epub ahead of print: 08 May 2018].

18 Calvaresi D, Marinoni M, Dragoni AF, et al. Real-Time multiagent systems for telerehabilitation scenarios. Artif Intell Med 2019;96:217-31.

19 Hasenohrl T, Windschnurer T, Dorotka R. Prescription of individual therapeutic exercises via smartphone APP for patients suffering from non-specific back pain a qualitative feasibility and quantitative pilot study. Wien Klin Wochen;132:115-23.

20 Levac D, Dumas HM, Meleis W. A tablet-based interactive movement tool for pediatric rehabilitation: development and preliminary usability evaluation. JMIR Rehabil Assist Technol 2018;5:e10307.

21 Paneroni M, Colombo F, Papalia A, et al. Is Telerehabilitation a safe and viable option for patients with COPD? A feasibility study. COPD 2015;12:217-25.

22 Giesbrecht EM, Miller WC. Effect of an mHealth wheelchair skills training program for older adults: a feasibility randomized controlled trial. Arch Phys Med Rehabil 2019;100:2159-66.

23 Grau-Pellicer M, Lalanza JF, Jovell-Fernández E, et al. Impact of mHealth technology on adherence to healthy PA after stroke: a randomized study. Top Stroke Rehabil 2020;27:354-68.

24 Gondim ITGdeO, Lins CCdosSA, Asano NMJ, et al. Individualized guidance and telephone monitoring in a self-supervised home-based physiotherapeutic program in Parkinson. Fisioterapia em Movimento 2017;30:559-68.

25 Venkataraman K, Morgan M, Amis KA, et al. Tele-assessment of the Berg balance scale: effects of transmission characteristics. Arch Phys Med Rehabil 2017;98:659-64.

26 Amatya B, Galea MP, Kesselring J, et al. Effectiveness of telerehabilitation interventions in persons with multiple sclerosis: a systematic review. Mult Scler Relat Disord 2015;4:358-69.
27 Block VAJ, Pitsch E, Tahir P, et al. Remote physical activity monitoring in neurological disease: a systematic review. PLoS One 2016;11:e0154335

28 Mani S, Sharma S, Omar B, et al. Validity and reliability of Internetbased physiotherapy assessment for musculoskeletal disorders: a systematic review. J Telemed Telecare 2017;23:379-91.

29 AHPScot. A guide to musculoskeletal (MSK) digital health consultations during coronavirus (COVID-19), 2020NHS Scotland. Available: https://ahpscot.wordpress.com/2020/04/22/a-guideto-musculoskeletal-msk-digital-health-consultations-duringcoronavirus-covid-19/ [Accessed 10/08/21].

$30 \mathrm{NI}$ Health and Social Care Board. AHP virtual consultation guidance, 2020. Available: https://view.pagetiger.com/coiyugy/1 [Accessed $10 / 08 / 21]$.

31 Teleswallowing Ltd. Telehealth service delivery guide, 2020. Available: https://www.rcslt.org/wp-content/uploads/media/ Teleswallowing-delivery-guide-2020.pdf?la=en\&hash=2ACBB3FD B65E1FAB3C7C7B0DF2499E03CC5DC571 [Accessed 10/08/21]

32 Charted Society of Physiotherapy. Telephone guidance for musculoskeletal practice, 2020. Available: https://www.csp.org. uk/news/coronavirus/remote-service-delivery-options/telephoneguidance-msk-practice [Accessed 10/08/21].

33 Charted Society of Physiotherapy. Digital tools to support service delivery, 2020. Available: https://www.csp.org.uk/news/coronavirus/ remote-service-delivery-options/digital-tools-support-servicedelivery [Accessed 10/08/21]

34 Charted Society of Physiotherapy. COVID-19: guide for rapid implementation of remote consultations, 2020. Available: https:// www.csp.org.uk/system/files/publication files/Remote\% 20consultations\%20top\%20tips\%20v10_1.pdf [Accessed 10/08/21].

35 Royal College of Occupational Therapists. Virtual Assessments/ Reviews: the script, 2020. Available: https://www.rcot.co.uk/practiceresources/occupational-therapy-topics/digital-first-occupationaltherapy [Accessed 12/10/20].

36 Royal College of Speech and Language Therapists. Maintaining security for telehealth sessions, 2020. Available: https://www.rcsit. org/wp-content/uploads/media/docs/Telehealth/INFO04RCSLTInfographic. pdf?la=en\&hash=D15EB355BC74DB59944CD3474EC4 E75E87AABD80 [Accessed 18/08/20].

37 East Lancashire Hospitals NHS Trust. Telepractice dysphagia assessmentsGuidelines for use with a patient in their own home/ nursing home, 2020. Available: https://www.rcslt.org/wp-content/ uploads/media/docs/Telehealth/03PDF-flow-chart-for-telepracticehome.pdf?la=en\&hash=3BC89B4762850483D842388D37AD4C6E 68B71C4D [Accessed 18/08/20].

38 Seuren LM, Wherton J, Greenhalgh T, et al. Physical examinations via video for patients with heart failure: qualitative study using conversation analysis. J Med Internet Res 2020;22:e16694.

39 Shaw SE, Seuren LM, Wherton J, et al. Video consultations between patients and clinicians in diabetes, cancer, and heart failure services: linguistic ethnographic study of Video-Mediated interaction. J Med Internet Res 2020;22:e18378.

40 Kuek A, Hakkennes S. Healthcare staff digital literacy levels and their attitudes towards information systems. Health Informatics $J$ 2020;26:592-612.

41 Poncette A-S, Glauert DL, Mosch L, et al. Undergraduate medical competencies in digital health and curricular module development: mixed methods study. J Med Internet Res 2020;22:e22161.

42 Shaw J, Shaw S, Wherton J, et al. Studying scale-up and spread as social practice: theoretical introduction and empirical case study. $J$ Med Internet Res 2017;19:e244.

43 Palacholla RS, Fischer N, Coleman A, et al. Provider- and patientrelated barriers to and facilitators of digital health technology adoption for hypertension management: Scoping review. JMIR Cardio 2019;3:e11951-e51.

44 Majeed A, Maile EJ, Coronini-Cronberg S. Covid-19 is magnifying the digital divide, 2020. BMJ opinion. Available: https://laptrinhx. $\mathrm{com} /$ news/covid-19-is-magnifying-the-digital-divide-pGZbpeA/amp/ [Accessed 10/08/21].

45 Hosseiniravandi M, Kahlaee AH, Karim H, et al. Home-based telerehabilitation software systems for remote supervising: a systematic review. Int J Technol Assess Health Care 2020;36:113-25.

46 Peretti A, Amenta F, Tayebati SK, et al. Telerehabilitation: review of the state-of-the-art and areas of application. JMIR Rehabil Assist Technol 2017;4:e7.

47 Aderonmu JA. Emerging challenges in meeting physiotherapy needs during COVID-19 through telerehabilitation. Bulletin of Faculty of Physical Therapy 2020;25:16. 